\title{
Discussion of "Turbulence Measurements with Acoustic Doppler Velocimeters" by Carlos M. García, Mariano I. Cantero, Yarko Niño, and Marcelo H. García
}

December 2005, Vol. 131, No. 12, pp. 1062-1073.

DOI: $10.1061 /(A S C E) 0733-9439(2005) 31: 12(1062)$

\author{
Bahareh Doroudian ${ }^{1}$; David Hurther ${ }^{2}$; and \\ Ulrich Lemmin ${ }^{3}$ \\ ${ }^{1}$ Research Assistant, LHE—ENAC, Ecole Polytechnique Fédérale de \\ Lausanne, St. 18, CH-1015 Lausanne, Switzerland. E-mail: \\ bahareh.doroudian@epfl.ch \\ ${ }^{2}$ Associate Scientist, LEGI-CNRS, Grenoble, France. \\ ${ }^{3}$ Research Associate, LHE-ENAC, Ecole Polytechnique Fédérale de \\ Lausanne, St. 18, CH-1015 Lausanne, Switzerland.
}

The paper by Garcia et al. deals with the problem of correctly measuring turbulence parameters with acoustic Doppler velocimeters (ADV; trade names ADV for Sontek and NDV for Nortek). The authors focus on the effects of sampling frequency and Doppler noise on turbulence parameters. To avoid loss of turbulence information, they suggest that data should be sampled above a determined frequency. In addition, noise should be removed by estimating the noise contribution. Their approach is based on a model-derived procedure. First, it would have been of interest to compare the modeled spectra with those obtained from their measurements to validate their model and instrument assumptions for the flow cases discussed. Second, the deviation from the $-5 / 3$ slope in the measured spectra due to filtering and/or noise effects has not been highlighted.

We investigated the authors' conclusions using a Vectrino (Nortek) ADV. Different from their instruments, a Vectrino has four receivers symmetrically spaced around the central emitter. The applied sampling frequencies, the relative position, and the size of the measuring volume, however, were identical to the NDV. Using four receivers allows measuring the vertical velocity component simultaneously in the two planes. This configuration enables the direct estimation of noise effects so that suitable correction procedures such as the one proposed by Hurther and Lemmin (2001; hereinafter called HLP) can be applied. The HLP takes advantage of the redundancy of the vertical velocity obtained in the two instrument planes. 


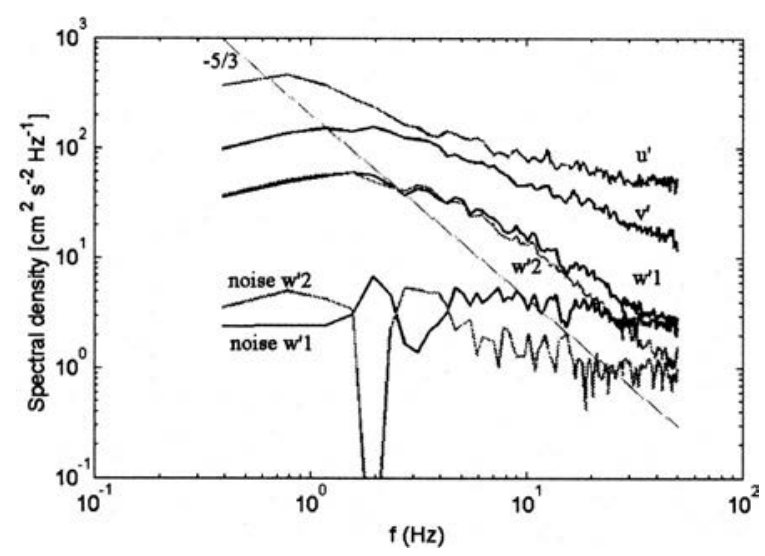

Fig. 1. Turbulence spectra of the $u, v$, and $w$ velocity components sampled at $100 \mathrm{~Hz}$; also shown are the noise spectra for the two vertical velocities

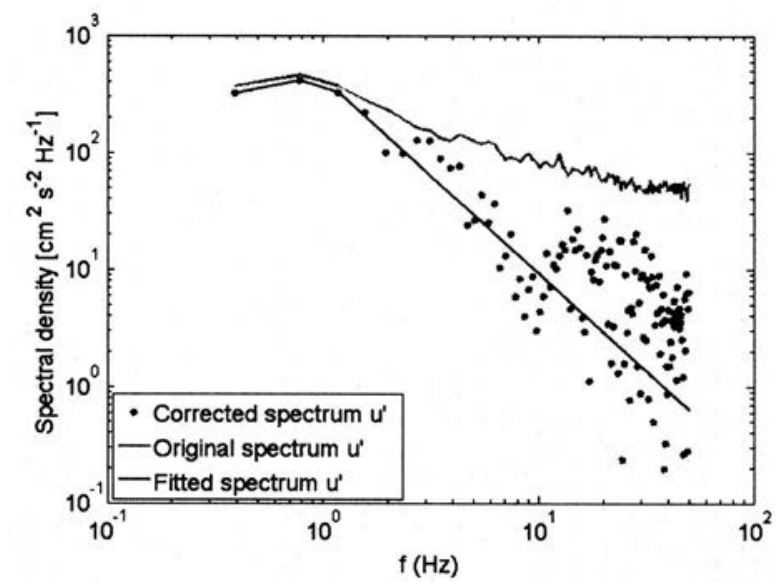

Fig. 2. Original, noise corrected, and fitted spectra for the longitudinal component sampled at $100 \mathrm{~Hz}$

It should be noted that Doppler noise is composed of several contributions that can be estimated (Garbini et al. 1982; Lhermitte and Lemmin 1990, 1994; Hurther and Lemmin 1998; Voulgaris and Trowbridge 1998) or eliminated (Garbini et al. 1982; Hurther and Lemmin 1998, 2001; Blanckaert and Lemmin 2006). For three receiver instruments, such as those used by the authors, the procedure proposed by Voulgaris and Trowbridge (1998) can be applied who emphasize that overestimates have to be expected. A sufficiently high sampling frequency is required for successful measurements.

Our measurements were carried out in an open channel at the LHE-EPFL. The channel is $0.60 \mathrm{~m}$ wide and $17 \mathrm{~m}$ long. The bottom was covered with a $0.1-\mathrm{m}$-thick gravel layer (size range $3-8 \mathrm{~mm} ; d_{50}=5.5 \mathrm{~mm}$ ). In this experiment, water depth and the measuring volume of the instrument were respectively $0.14 \mathrm{~m}$ and $0.05 \mathrm{~m}$ above the bed. The convective velocity is $0.6 \mathrm{~ms}^{-1}$. Data were recorded about $12 \mathrm{~m}$ from the channel entrance where turbulence is well developed for at least $3 \mathrm{~min}$ with sampling frequencies of $100,75,50,25$, and $10 \mathrm{~Hz}$. The instrument was mounted downward looking with one receiver plane oriented along the flow and the second one in the transversal direction. Two experiments were carried out. In the first one, mean values for correlation and SNR were about 84 and $24 \mathrm{~dB}$. In this experiment we used hydrogen bubbles as "seeding material" (Blanckaert and Lemmin 2006). For all sampling frequencies, the data appeared "clean" with only a few spikes. In the second experiment, mean values for correlation and SNR were 81 and $22 \mathrm{~dB}$ without any seeding procedure. Although these quality parameters were high, frequent spikes were observed, in particular in the longitudinal plane.

Following Nezu and Nakagawa (1993), $u, v$ and $w$ denote the velocity fluctuation and $u^{\prime}, v^{\prime}$, and $w^{\prime}$ denote the RMS values (turbulence intensities). For each of the sampling frequencies, the turbulence intensities and spectra of each velocity were calculated. The noise spectra were obtained using the HLP by calculating the cross spectrum between the two vertical components. The noise spectrum of the longitudinal component $u$ was determined as outlined in HLP and subtracted from the original spectrum of $u$. To fit a curve to the noise corrected spectrum (NCS), we kept the NCS at the low frequency end and curve fitted the NCS points starting where the $-5 / 3$ slope is established in the spectrum and ending at the Nyquist frequency. An estimate of the variance can be obtained by integrating the surface under the spectral curve. This was done for all three spectra resulting in $u_{\text {orig }}^{\prime}$ for the original spectrum, $u_{\text {cor }}^{\prime}$ for the NCS, and $u_{\text {fit }}^{\prime}$ for the fitted one.

Fig. 1 shows a typical result for the data sampled at $100 \mathrm{~Hz}$. As can be seen, both of the vertical velocities, $\left(w_{1}\right)$ the longitudinal plane and particularly $\left(w_{2}\right)$ in the transversal plane closely follow the $-5 / 3$ slope over an extended region. Both noise spectra are nearly flat indicating white noise. Fig. 2 shows spectra of the longitudinal components (original and NCS) as well as a fitted spectrum. Although we can see that the slope of the NCS in the midfrequency range is close to $-5 / 3$, at the high frequency end, the noise is not completely removed by the HLP method, resulting in significant scatter.

The aforementioned procedure was executed for all velocity components and sampling frequencies. The results for the longitudinal component are summarized in Table 1 . It can be seen that

Table 1. Estimates of the Three Turbulence Intensities (in $10^{-2} \mathrm{~ms}^{-1}$ ) for the Longitudinal Velocity Sampled at Different Frequencies

\begin{tabular}{|c|c|c|c|c|c|c|c|c|c|}
\hline \multirow{2}{*}{$\begin{array}{l}f \\
(\mathrm{~Hz})\end{array}$} & \multicolumn{3}{|c|}{$h=0.14 \mathrm{~m}(\exp 1)$} & \multicolumn{3}{|c|}{$\mathrm{h}=0.14 \mathrm{~m}(\exp 2)$} & \multicolumn{3}{|c|}{$\mathrm{h}=0.09 \mathrm{~m}$} \\
\hline & $u_{\text {orig }}^{\prime}$ & $u_{c o r}^{\prime}$ & $u_{\text {fit }}^{\prime}$ & $u_{\text {orig }}^{\prime}$ & $u_{c o r}^{\prime}$ & $u_{f i t}^{\prime}$ & $u_{\text {orig }}^{\prime}$ & $u_{c o r}^{\prime}$ & $u_{f i t}^{\prime}$ \\
\hline 100 & 8.83 & 4.89 & 4.69 & 15.16 & 8.66 & 6.16 & 4.69 & 3.74 & 3.74 \\
\hline 75 & 7.93 & 4.89 & 4.24 & 13.96 & 8.06 & 6.16 & 5.65 & 3.74 & 3.00 \\
\hline 50 & 7.81 & 4.89 & 4.69 & 11.31 & 6.92 & 5.47 & 5.19 & 3.74 & 3.00 \\
\hline 25 & 5.47 & 4.35 & 4.12 & 11.83 & 7.41 & 4.69 & 4.89 & 3.74 & 3.46 \\
\hline 10 & 4.89 & 4.24 & 4.00 & 7.21 & 5.65 & 5.00 & 5.65 & 4.12 & 2.82 \\
\hline
\end{tabular}


$u_{\text {orig }}^{\prime}$ decreases by a factor of almost two between the highest and the lowest sampling frequency. However, it decreases slower between 100 and $50 \mathrm{~Hz}$ than between 50 and $10 \mathrm{~Hz}$. The values for the NCS are by a factor of two lower than those of the original spectrum for $100 \mathrm{~Hz}$ and remain constant until $50 \mathrm{~Hz}$. They then drop by about $10 \%$ for $10 \mathrm{~Hz}$. This difference between the original data and the NCS confirms the observation by Lohrmann et al. (1994) that uncorrected data are biased to higher values. The values obtained from the fitted spectra show a difference of about $10 \%$ between the highest and the lowest frequency. In between, the variation is random.

The magnitude of the values from the different spectra indicates the importance of proper noise removal. Comparing $u_{\text {orig }}^{\prime}$ and $u_{\text {cor }}^{\prime}$, the decreasing tendency in $u_{\text {orig }}^{\prime}$ for decreasing sampling frequency can essentially be attributed to noise effects and not to filtering related to the sampling frequency. If filtering had been the dominant cause of the unresolved velocity scales in the higher spectral range, the slope of the spectra in this region would have been greater than the $-5 / 3$ value. This is not always the case for different sampling frequencies.

In this study, we documented the importance of proper noise removal. Considering the sampling frequency criteria suggested by Nezu and Nakagawa (1993), turbulence should not be sampled below $75 \mathrm{~Hz}$ in our case. However $u_{\text {cor }}^{\prime}$ remains constant down to $50 \mathrm{~Hz}$. In other words, our observation demonstrates that the Vectrino ADV is a robust instrument because it still produces reliable results well below that threshold value. Thus, when proper sampling criteria are respected, filtering due to change in sampling frequency has no effect on the results.

The curve fitting of the noise corrected spectrum was done to determine whether the uncorrected noise and aliasing may affect the estimates. For our results the difference is about 5\%. Considering that curve fitting is not an ideal procedure and that other undetermined uncertainties in the measuring procedure remain, this value appears acceptable and indicates that those deviations do not significantly affect the results.

We have applied this procedure to the second data set in which a fairly large number of spikes occurred. For the present analysis, we did not eliminate the spikes from the data set. These spikes may be due to random noise or aliasing. Although aliasing can be dealt with by using procedures modified from those suggested by Franca and Lemmin (2006), random noise is difficult to eliminate from the $u, v$, and $w$ velocity data. Furthermore, it has to be remembered that due to the system configuration, spikes in one velocity component may also affect the other components. Thus, spike removal procedures such those as suggested by Goring and Nikora (2002) have to be applied with caution. The Vectrino ADV allows for recording beam velocities instead of $u, v$, and $w$ velocities. This recording has a great advantage in that spikes can be removed individually from each beam time series before constructing the $u, v$, and $w$ velocities. This allows for a much more objective approach than previous ones (Goring and Nikora 2002).

The results in Table 1 indicate that the overall trends observed in the first data set are reproduced in the second one. However, the level of all values is roughly double that of the first data set. This shows that noise removal by the HLP cannot eliminate the effects of spikes and that spikes have a much more detrimental effect on the quality of the results than the sampling aspect previously mentioned. On the other hand, it appears from our results that sampling at low frequency would be the better strategy in this case. Taking the first data set as reference, the noise corrected and fitted results at low sampling frequencies in the spiked data are closest to those observed in the first data set at frequencies above the threshold level.

In a final test we applied the HLP to a data set taken in the same channel in a flow of $0.09 \mathrm{~m}$ water depth and a convective velocity of $0.32 \mathrm{~ms}^{-1}$, which is about $50 \%$ of the convective velocity in the experiments above. Again, we used hydrogen bubble seeding. Results in Table 1 show that the original spectra vary randomly. Thus there is no filtering effect related to the sampling frequency. This is even more obvious in the NCS, which remains constant down to $25 \mathrm{~Hz}$. The fitted data which depend on the indication of $a-5 / 3$ trend in the spectrum show poor results for the $10 \mathrm{~Hz}$ case. Overall these data indicate once more that apart from spike removal, noise removal is the most important process for increasing the reliability of the data.

Our analysis has shown that the recommendations and conclusions by the authors cannot be considered as a universal guide when making turbulence measurements with ADVs. Our investigation has demonstrated that four-receiver ADV instruments, such as the Vectrino, open up new ways to treat data that lead to greatly improved results in turbulent flows. This suggests that using modern ADV instrumentation, turbulence studies can be carried out along the following procedure:

- Ensure that the flow has sufficient scattering targets. Wherever seeding is needed, hydrogen bubble seeding (Blanckaert and Lemmin 2006) has proven to give excellent results in large channel installations where injection of small particles is technically and economically not feasible.

- Record data as beam velocities at sampling frequencies near and preferably above the threshold level as indicated by Nezu and Nakagawa (1993). This allows for subsequent spike removal by de-aliasing procedures (Franca and Lemmin 2006) or data splicing such as spline procedures over adjacent points.

- Transform beam velocities into $u, v$, and $w$ velocities and apply noise removal procedures such as HLP (Hurther and Lemmin 2001). The noise removal procedure can be further extended as suggested by Blanckaert and Lemmin (2006).

\section{References}

Blanckaert, K., and Lemmin, U. (2006). "Means of noise reduction in acoustic turbulence measurements." J. Hydraul. Res., 44(1), 3-17.

Franca, M., and Lemmin, U. (2006). "Eliminating velocity aliasing in acoustic Doppler velocity profiler data." Meas. Sci. Technol., 17(2), 313-322.

Garbini, J. L., Forster, F. K., and Jorgensen, J. E. (1982). "Measurement of fluid turbulence based on pulsed ultrasound techniques. Part 1: Analysis." J. Fluid Mech., 118, 445-470.

Goring, D. G., and Nikora, V. I. (2002). "Despiking acoustic Doppler velocimeter data." J. Hydraul. Eng., 128(1), 117-126.

Hurther, D., and Lemmin, U. (1998). "A constant beam width transducer for 3D acoustic Doppler profile measurements in open channel flows." Meas. Sci. Technol., 9(10), 1706-1714.

Hurther, D., and Lemmin, U. (2001). "A correction method for turbulence measurements with a 3D acoustic Doppler profiler." J. Atmos. Ocean. Technol., 18(3), 446-458.

Lhermitte, R., and Lemmin, U. (1990). "Probing water turbulence by high frequency Doppler sonar." Geophys. Res. Lett., 17(10), 15491552.

Lhermitte, R., and Lemmin, U. (1994). "Open-channel flow and turbulence measurements by high resolution Doppler sonar." J. Atmos. Ocean. Technol., 11(5), 1295-1308.

Lohrmann, A., Cabrera, R., and Kraus, N. (1994). "Acoustic Doppler velocimeter (ADV) for laboratory use.” Proc., Symp. on Fundamentals and Advances in Hydraulic Measurements and Experimentation, 
ASCE, New York, 351-365.

Nezu, I., and Nakagawa, H. (1993). Turbulence in open channel flows, IAHR, Balkema, Rotterdam, The Netherlands.

Voulgaris, G., and Trowbridge, J. H. (1998). "Evaluation of the acoustic Doppler velocimeter (ADV) for turbulence measurements." J. Atmos. Ocean. Technol. Vol. 15, 272-289. 